\title{
RESONANCE p:p+q IN SATELLITE ORBITS
}

\author{
PEDRO R. GROSSO AND WAGNER SESSIN \\ Depto. de Mecânica do Vôo e Orbital \\ Instituto Tecnológico de Aeronáutica \\ 12225, São José dos Campos, SP, Brasil
}

\begin{abstract}
In this paper we consider the motion of a satellite whose mean motion is commensurable with the frequency of the rotational motion of the planet in the ratio $p: p+q$.
\end{abstract}

\section{Resonance $p: p+q$}

In the present work we study the motion of a satellite around an oblate planet. It is supposed that the mean motion of the satellite orbit is commensurable with the frequency of the rotational motion of the planet.

The gravitational potential of an oblate planet that acts on a satellite can be written in the form (Kaula, 1966)

$$
R=\sum_{l=2}^{\infty} \sum_{m=0}^{l} \sum_{s=0}^{r} \sum_{r=-\infty}^{\infty} R_{l m s r}
$$

where

$$
R_{l m s r}=\left(\frac{\mu}{a}\right)\left(\frac{a_{e}}{a}\right)^{l} F_{l m s}(i) G_{l s r}(e) J_{l m} \begin{cases}\cos \Psi_{l m s r} & \text { if } l-m \text { even } \\ \sin \Psi_{l m s r} & \text { if } l-m \text { odd }\end{cases}
$$

and

$$
\Psi_{l m s r}=(l-2 s) \omega+(l-2 s+r) M+m\left(\Omega-n_{0} t-\lambda_{l m}\right) .
$$

$(a, e, i, M, \omega, \Omega)$ are the Keplerian elements of the satellite's orbit, $J_{l m}$ are dimensionless coefficients, $\mu$ is the Gaussian constant, $a_{e}$ is the mean equatorial radius of the planet, $F_{l m},(i)$ is the inclination function, and, $G_{l s r}(e)$ is eccentricity function.

The resonance effects arise when

$$
p \dot{M}-(p+q)\left(n_{0}-\dot{\Omega}\right)+(p-r) \dot{\omega} \simeq 0
$$

where $p, q$ and $r$ are integers.

The resonance condition given by Equation (2) occurs due to the $p: p+q$ commensurability and appears in many harmonics in the disturbing function. In this work we assume that there is a dominant critical term which is identified by a value of $r$, therefore only one critical harmonic is taken into account. If we suppose that $r_{c}$ is the value of $r$ that determines this critical harmonic then it is factored by

$$
e^{\left|r_{c}\right|}\left(\sin \frac{i}{2}\right)^{\left|r_{c}+q\right|}
$$

The periodic terms, except the resonant one, are eliminated. The dynamical system is reduced to one degree of freedom; therefore, completely integrable. In order 
to study the resonant system we expand the hamiltonian in the neighborhood of the exact resonance, and only lower order terms are maintaining in the hamiltonian. The solutions of the differential equations depend on the order of the resonance and on the critical term assumed as dominant (Grosso,1989). Once $q$ defines the order of the resonance and $r_{c}$ identifies the critical term, the solutions depend on the order of the eccentricity and inclination that factored the critical term according with Equation (3). More precisely, it depends on the value of

$$
\nu=\left|r_{c}\right|+\left|r_{c}+q\right| \text {. }
$$

For each value of $\nu$ different topological structure of the phase space is obtained.

\section{Discussion}

The topological structures for resonances for different values of $\nu$ has been studied and they are similar to that obtained for the problem of restricted circular three body (Lemaître, 1984). For resonances of the same orders (fixed value of $q$ ), it has been considered others harmonics simultaneously and different topological structures has been obtained.

\section{Acknowledgements}

This work was partially sponsored by CNPq. The authors thanks to FAPESP by the financial aids.

\section{References}

Grosso,P.R.:1989, Movimento Orbital Perturbado de um Satélite Artificial em Ressonância 2:1, M.Sc. Thesis, ITA, São José dos Campos.

Kaula,W.M.:1966, Theory of Satellite Geodesy, Blaisdell Publishing Company, Massachusetts. Lemaitre,A.:1984, Celest. Mech. 32, 109. 